\title{
Train operation minimizing energy consumption in DC electric railway with on-board energy storage device
}

\author{
K. Matsuda, H. Ko \& M. Miyatake \\ Sophia University, Japan
}

\begin{abstract}
The optimal train operation which minimizes sum of supplied energy from substations is presented in this paper. In recent years, the energy storage devices have enough energy and power density to use in trains as on-board energy storage. The electric double layer capacitor (EDLC) is assumed as an energy storage device in our study, because of its high power density. The on-board storage can assist the acceleration/deceleration of the train and may decrease energy consumption. Many works on the application of the energy storage devices to trains were reported, however, they did not deal enough with the optimality of the control of the devices. On the other hand, our previous works were to optimize acceleration/deceleration commands of the train for minimizing energy consumption without the energy storage device. Therefore, we intend to optimize acceleration/deceleration commands together with current commands through energy storage devices as our next research target. The proposed method can determine the optimal acceleration/deceleration and current commands at every sampling point. For this purpose, the optimal control problem of the train operation is formulated mathematically. It is generally difficult to solve the problem because the problem is composed of a large-scale non-linear system. However, the Sequential Quadratic Programming (SQP) can be applied to solve the problem. Two results with and without on-board energy storage device are compared. These optimized results indicate that the total energy consumption is reduced by at least $0.35 \%$ by using the EDLC. The relation between internal resistance and energy consumption is also revealed.
\end{abstract}

Keywords: electric double layer capacitor (EDLC), optimal control, energy saving operation, SQP method. 


\section{Introduction}

In recent years, the energy storage devices have enough energy and power density to use in trains as on-board energy storage. The devices are for instance, a secondary battery and an Electric Double Layer Capacitor (EDLC). Above all, the EDLC has advantages such as maintenance free, long lifetime, rapid charge/discharge with large current and high efficiency. Therefore, the EDLC is the most suitable to equip trains as an auxiliary power supply. The on-board EDLC is useful because of the following two reasons. Firstly, it decreases the loss of circuit resistance by compensating voltage drop. Secondly, it enables us to utilize and recycle regenerative power efficiently and prevent regenerative failure.

Many works on the application of the energy storage devices to trains were reported. However, from an energy-saving point of view, they did not deal enough with the optimality of the control of the devices. On the other hand, our previous works $[1,2]$ was to optimize notch commands which determine the acceleration/deceleration force in the train without energy storage devices. We optimize notch commands together with charge/discharge commands with making use of the experience of our previous study. It is significant to investigate the optimal charge/discharge command minimizing energy consumption in order to maximize the effect of installing the EDLC.

In this paper, we intend to formulate the optimal control problem of the train operation to find notch and charge/discharge commands which minimize amount of consumed energy, propose how to solve it, discuss the optimized results and find knowledge of the optimal operation. The knowledge will be applied to the future charge/discharge controllers for EDLCs.

\section{Modeling of DC feeding Circuit}

We modeled a DC feeding circuit when there is only one train between substations. The model circuit appears in fig. 1 . In this figure, $V_{s}$ and $R_{0}$ are the supply voltage and the internal resistance at a substation respectively. The values of $R_{1}$ and $R_{2}$ are wire resistances. These resistance values are proportional to the distance between the train and substation position. Positions of substations and stations are shown in fig. 2. The constants $C$ and $R_{c}$ are the capacitance and internal resistance in the capacitor respectively.

It is necessary to convert voltage by using a chopper because the voltage difference is high between the pantograph and capacitor. The chopper characteristic is too complicated to be examined in detail here. Therefore, we solved the circuit equation on the assumption that the chopper efficiency is $95 \%$.

In addition, the energy consumption in the train is regarded as constant in short time because acceleration/deceleration commands do not change often. The motor-inverters of the train are modeled as a current load that helps solving circuit equations simply. 


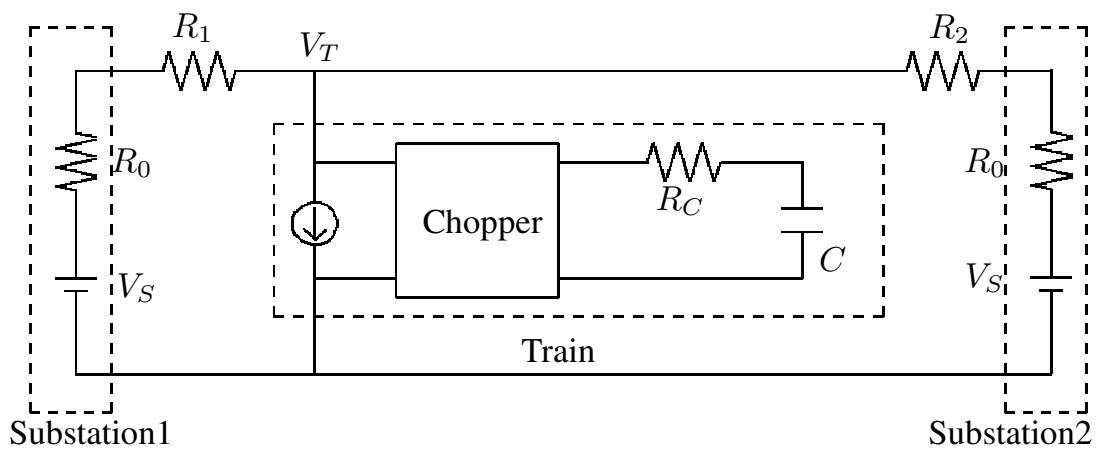

Figure 1: Circuit model with one train between substations.

\begin{tabular}{|l|l|l|l|l|l|l|l}
$\mathrm{SS} 1$ & $L_{a}$ & \multirow{3}{*}{$\mathrm{DS}$} & $L$ & $L_{b}$ & $\mathrm{AS} 2$ & $\begin{array}{l}\text { DP:departure station } \\
\text { AS:arrival station } \\
\text { SS:substation }\end{array}$
\end{tabular}

Figure 2: Positions of stations and substations.

\section{Formulation of optimal control problem}

We formulated the optimal control problem in this section. Here, variables are defined as follows. Control inputs $n$ and $u$ determine the acceleration/deceleration force and charge/discharge current through the capacitor, respectively. They are defined as table 1 . State variables $x, v$ and $V_{c}$ indicate the train position, speed and capacitor voltage, respectively. Variable $V_{T}$ is the voltage at the pantograph. In fact, it is a state variable if control inputs are determined and the circuit equation can be solved. However, we defined $V_{T}$ as the auxiliary variable because it is difficult to solve circuit equations analytically. Additionally, these all variables depend on time $t$. The optimal control problem is described as follows, mathematically.

Table 1: Definition of control inputs $n$ and $u$.

\begin{tabular}{|l|l|l|}
\hline$n$ or $u$ & Operation mode & Current through the capacitor \\
\hline-1 & maximum deceleration & maximum charge \\
negative & deceleration & charge \\
0 & coast & wait \\
positive & acceleration & discharge \\
1 & maximum acceleration & maximum discharge \\
\hline
\end{tabular}


Minimizing the objective function

$$
J=\int_{0}^{T} V_{s} I_{s}\left(x, V_{T}\right) d t
$$

Subject to the following equality and inequality constraints

$$
\begin{aligned}
& \dot{x}=v \\
& \dot{v}=f\left(n, v, V_{T}\right)-r(v) \\
& \dot{V}_{c}=-I_{c}(u) / C \\
& P_{T}\left(n, v, V_{T}\right)=P_{s}\left(x, V_{T}\right)+P_{c}\left(u, V_{c}\right) \\
& x(0)=0 v(0)=0 \quad V_{c}(0)=V_{c_{-} f \text { irst }} \\
& x(T)=L v(T)=0 \quad V_{c}(T)=V_{c_{-} f \text { inal }} \\
& -1 \leq n \leq 1 \\
& -1 \leq u \leq 1 \\
& V_{T \_ \text {min }} \leq V_{T} \leq V_{T \_ \text {max }} \\
& V_{c_{-} \text {min }} \leq V_{c} \leq V_{c_{-} \text {max }} \\
& 0 \leq x \leq L \\
& v \geq 0
\end{aligned}
$$

where

$I_{s}$

$I_{c}$

$f, r$

$P_{T}$

$P_{s}, P_{c}$

sum of load currents supplied by substations

current through the capacitor

$V_{T \_m i n}, V_{T \_ \text {max }}$ acceleration/deceleration force and running resistance per $\mathrm{kg}$

$V_{c \_ \text {min }}, V_{c \_ \text {max }}$ electric power supplied to motor-inverters of the train

$V_{c_{-} \text {first }}, V_{c_{-} \text {final }}$ power from substations and the capacitor

$L, T$

lower and upper limitations of the voltage at the pantograph

lower and upper limitations of the capacitor voltage

first and final values of the capacitor voltage distance and running time between the departure and arrival station.

The objective function is sum of supplied energy by two substations given as eqn. (1). Equality constraints are given as eqns. (2-7). Eqns. (2),(3) are motion equations of the train. The capacitor voltage is given as the eqn. (4). As mentioned above, we must solve the circuit eqn. (5) because we defined $V_{T}$ as an auxiliary variable. Eqns. (6),(7) describe the initial and final conditions of state variables. Inequality constraints of control inputs, state and auxiliary variables are shown in eqns. (8-13). Especially, we did not consider speed limitations in eqn. (13). 
We defined functions as below.

$$
\begin{aligned}
& P_{T}\left(n, v, V_{T}\right)= \begin{cases}M v f\left(n, v, V_{T}\right) m_{e} & (n \geq 0) \\
\operatorname{Mvf}\left(n, v, V_{T}\right) / g_{e} & (n \leq 0)\end{cases} \\
& P_{s}\left(x, V_{T}\right)=\left(\begin{array}{ll}
\left.\frac{V_{s}-V_{T}}{R_{0}+R_{1}(x)}+\frac{V_{s}-V_{T}}{R_{0}+R_{2}(x)}\right)
\end{array}\right. \\
& R_{1}(x)=\left(L_{a}+x\right) r_{0} \quad R_{2}(x)=\left(L-x+L_{b}\right) r_{0}
\end{aligned}
$$

Here, $m_{e}$ and $g_{e}$ are motor/generator efficiency. Wire resistances $R_{1}$ and $R_{2}$ are given in eqn. (16) when the position of the departure station is defined as $x=0$. The constants $L_{a}$ and $L_{b}$ indicate the distance from the departure and arrival station to the substation 1 and substation 2 shown in fig. 1 . The constant $r_{0}$ is the wire resistance per meter. The constant $c_{e}$ is the chopper efficiency. The constant $I_{c_{-} \max }$ is the rated value of the current from the capacitor.

Additionally, maximum acceleration/deceleration characteristics, such as the control input $n$ is 1 or -1 , and running resistance are given in fig. 3 . these characteristics are influenced by the voltage at the pantograph $V_{T}$. Especially, we assume that the braking system is the air supplement control. In short, the use of electrical and mechanical blended braking system is considered if the regenerative braking force is not enough for the specific braking force. Moreover, we are not concerned with the characteristic of the squeezing control because we also assume the regenerative power can be absorbed at substations.

The absorbed power can be accounted in eqn. (1).
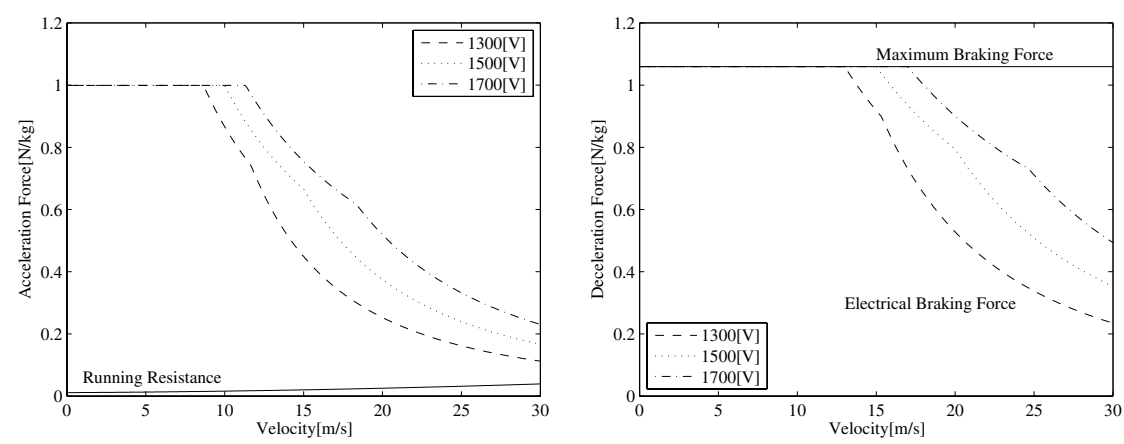

Figure 3: Acceleration/deceleration characteristics and running resistance. 


\section{Optimization method}

We show the optimization method for solving the optimal control problem. The optimal control problem is solved by Sequential Quadratic Programming (SQP). $\mathrm{SQP}$ is an optimization method to solve a general nonlinear programming problem. A general optimal control problem with equality and inequality constraints can be written as

$$
\begin{array}{lll}
\text { Minimize : } & J(\boldsymbol{\omega}) \\
\text { Subject to : } & g_{i}(\boldsymbol{\omega})=0 & \left(i=1, \cdots, n_{g}\right) \\
& h_{j}(\boldsymbol{\omega}) \leq 0 \quad\left(j=1, \cdots, n_{h}\right)
\end{array}
$$

where $\boldsymbol{\omega}=\left(\mathbf{n}, \mathbf{u}, \mathbf{V}_{\mathbf{T}}, \mathbf{x}, \mathbf{v}, \mathbf{V}_{\mathbf{c}}\right)^{\top}$ is the vector of variables, $J$ is the objective function, $g$ and $h$ are equality and inequality constraints, $n_{g}$ and $n_{h}$ are the number of equality and inequality constraints.

Next, the objective function is expressed using the second order approximation around the feasible point $\boldsymbol{\omega}^{(k)}$. Similarly, equality and inequality constraints are also expressed as the first order approximation around the feasible point $\boldsymbol{\omega}^{(k)}$ in problem (19). The transformed problem is shown as

$$
\begin{array}{lll}
\text { Minimize : } & \frac{1}{2} \mathbf{d}_{k}^{\top} \mathbf{B}_{k} \mathbf{d}_{k}+\nabla J\left(\boldsymbol{\omega}^{(k)}\right) \mathbf{d}_{k} \\
\text { Subject to : } & g_{i}\left(\boldsymbol{\omega}^{(k)}\right)+\nabla g_{i}\left(\boldsymbol{\omega}^{(k)}\right) \mathbf{d}_{k}=0 \quad\left(i=1, \cdots, n_{g}\right) \\
& h_{j}\left(\boldsymbol{\omega}^{(k)}\right)+\nabla h_{j}\left(\boldsymbol{\omega}^{(k)}\right) \mathbf{d}_{k} \leq 0 \quad\left(j=1, \cdots, n_{h}\right)
\end{array}
$$

where $\mathbf{d}_{k}=\boldsymbol{\omega}-\boldsymbol{\omega}^{(k)}, \mathbf{B}_{k}$ is positive definite matrix. In general, the problem (20) can be solved by the interior point method $[3,4]$. The optimization result of the problem (20) is a search direction $\mathbf{d}_{k}$.

Here, we define merit function as

$$
\psi(\boldsymbol{\omega})=J(\boldsymbol{\omega})+\mu\left[\sum_{i=1}^{n_{g}}\left|g_{i}(\boldsymbol{\omega})\right|+\sum_{i=1}^{n_{h}} \max \left(h_{j}(\boldsymbol{\omega}), 0\right)\right]
$$

where $\mu$ is a large positive constant. Finally, we have to find $\alpha$ to minimize $\psi\left(\boldsymbol{\omega}^{(k+1)}\right)$ where $\boldsymbol{\omega}^{(k+1)}=\boldsymbol{\omega}^{(k)}+\alpha \boldsymbol{d}_{k}$. The vector $\boldsymbol{\omega}^{(k+1)}$ is the next feasible point. Consequently, The optimization problem (19) can be solved by iterating the following procedure.

Step 1) give the initial feasible point $\boldsymbol{\omega}^{(0)}$, and set $k=0$

Step 2) solve problem (20), and obtain a search direction $\mathbf{d}_{k}$

Step 3) stop iteration if the norm $\left\|\mathbf{d}_{k}\right\|$ is less than $10^{-6}$.

Step 4) find $\alpha$ minimizing the merit function, and obtain the next feasible point $\omega^{(k+1)}$

Step 5) set $k=k+1$, and return step2 


\section{Optimization result}

Optimization results are presented in this section. Specific parameters are presented as table 2 . Here, the continuous time formulation must be transformed to a discrete time program in order to apply SQP to the optimal control problem. Therefore, $\Delta t$ is defined as the sampling interval. In addition, the final capacitor voltage is given to equal the initial one. So, the capacitor does not have to be charged when the train arrives at the station.

We show three optimization results when the train run on straight line without speed limitations and gradients. Case 1 is the optimization result of the train without the capacitor. Case 2 and Case 3 show results of the sensitive analysis in case of the constant $R_{c}$ is $0.3[\Omega]$ or $0.03[\Omega]$ respectively. These three optimization results are shown in table 3 .

Case 1: The optimization result is shown in fig. 4. The optimal train operation consists of the maximum acceleration, reduced acceleration by degrees, coasting and maximum deceleration. In previous works $[1,2]$, we obtained the similar optimization result. Therefore, these results indicate the reliability of the proposed method.

Case 2: fig. 5(a) shows the optimization result in case that $R_{c}$ is $0.3[\Omega]$. The optimal control input $n$ do not differ from one in case 1 . Next, we examine charge/discharge characteristics of the capacitor. As supplied power to the train is higher, discharge current is higher. Similarly, this pattern of the charge characteristic is also represented when the train decelerates. Qualitatively, this

Table 2: Specific parameters.

\begin{tabular}{|c|c|c|c|}
\hline \multicolumn{2}{|c|}{ Operating condition } & \multicolumn{2}{|c|}{ capacitor parameters } \\
\hline$T, \Delta t$ & $130[\mathrm{~s}], 1[\mathrm{~s}]$ & $C$ & $32.3[\mathrm{~F}]$ \\
$L$ & $2000[\mathrm{~m}]$ & $V_{c \_ \text {max }}$ & $560[\mathrm{~V}]$ \\
$R_{0}$ & $0.03[\Omega]$ & $V_{c \_ \text {min }}$ & $300[\mathrm{~V}]$ \\
$V_{s}$ & $1500[\mathrm{~V}]$ & $V_{c_{-} \text {first }}$ & $560[\mathrm{~V}]$ \\
$r_{0}$ & $0.04 \times 10^{-3}[\Omega / \mathrm{m}]$ & $V_{c_{-} \text {final }}$ & $560[\mathrm{~V}]$ \\
$L_{a}, L_{b}$ & $5000[\mathrm{~m}]$ & $I_{c_{-} \text {max }}$ & $500[\mathrm{~A}]$ \\
$M$ & $250 \times 10^{3}[\mathrm{~kg}]$ & $R_{c}$ & $0.3[\Omega]$ or $0.03[\Omega]$ \\
\hline
\end{tabular}

Table 3: Optimization results.

\begin{tabular}{|l|c|c|}
\hline & Total energy consumption [MJ] & Energy-saving effect[\%] \\
\hline Case 1 & 27.55 & - \\
Case 2 & 27.45 & $0.35 \%$ \\
Case 3 & 26.74 & $2.92 \%$ \\
\hline
\end{tabular}


characteristic is proper, because it is the most effective control to compensate the voltage drop at the pantograph and prevent the regenerative failure. In Case 2, despite the lower limit value of the capacitor voltage $V_{c_{-} \min }$ set to $300[\mathrm{~V}]$, the capacitor stops discharging when the value of the capacitor voltage drops to about $480[\mathrm{~V}]$. This result is attributed to the higher interior resistance of the capacitor. As a result, the efficiency of the capacitor itself is severely down according to the voltage drop of the capacitor.

Case 3: The optimization result is shown as fig. 5(b). In this case, there is also little variation in control input $n$. However, the optimal charge/discharge command includes two significant difference compared to Case 2. Firstly, the capacitor voltage reaches the value of the lower limitation when control input $n$ changes from acceleration to coast at about time 50[s]. Secondly, substations supply the power with a small current for charging the capacitor when the train coasts. It is found from the result that the capacitor is utilized effectively. Finally, the total energy consumption is $26.74[\mathrm{MJ}]$. Compared with Case 1 , the total energy consumption is reduced about $2.92 \%$. The result indicates that energy-saving effect is higher if the interior resistance of the capacitor is lower in the future.
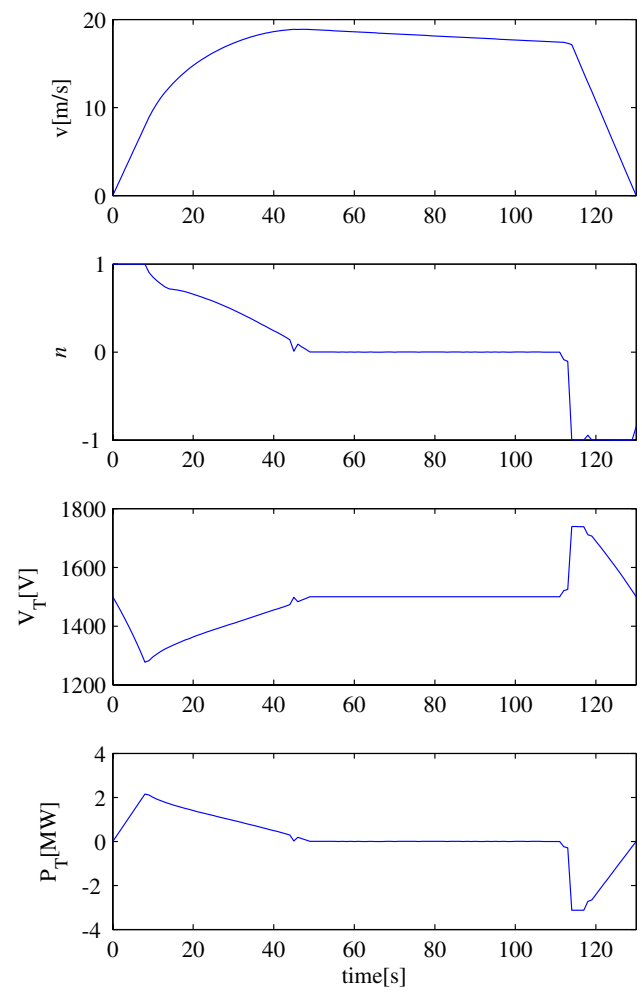

Figure 4: Optimization result (case 1). 


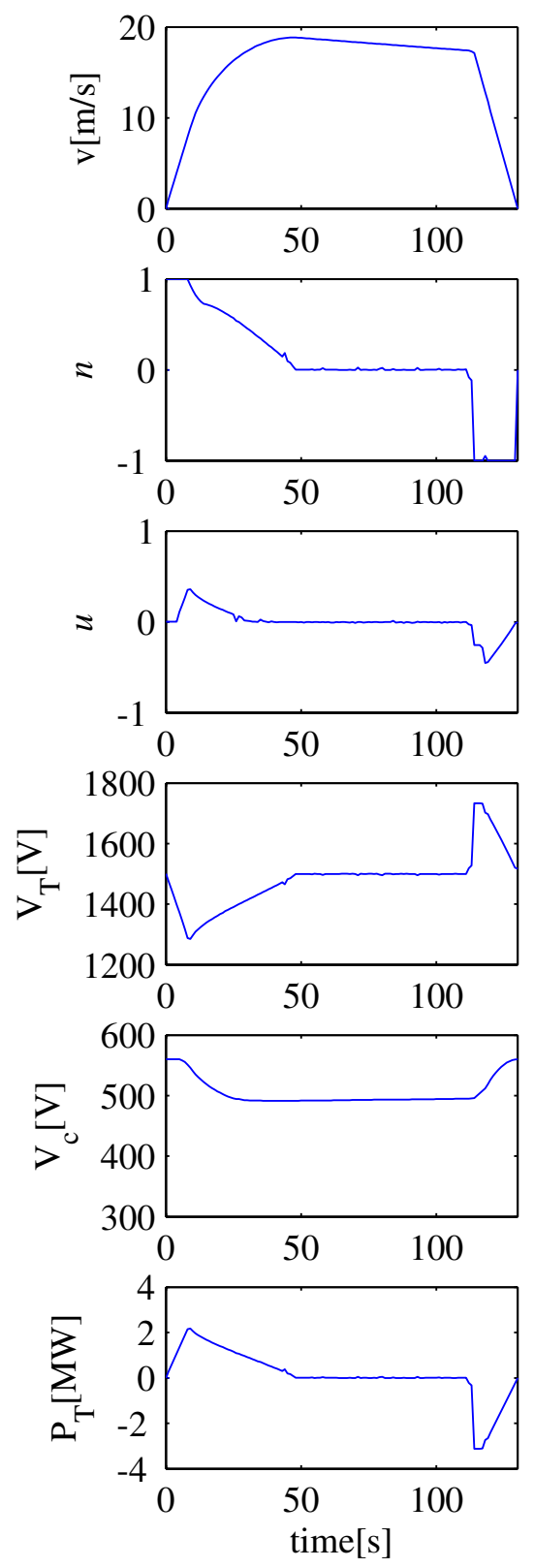

(a)Case 2
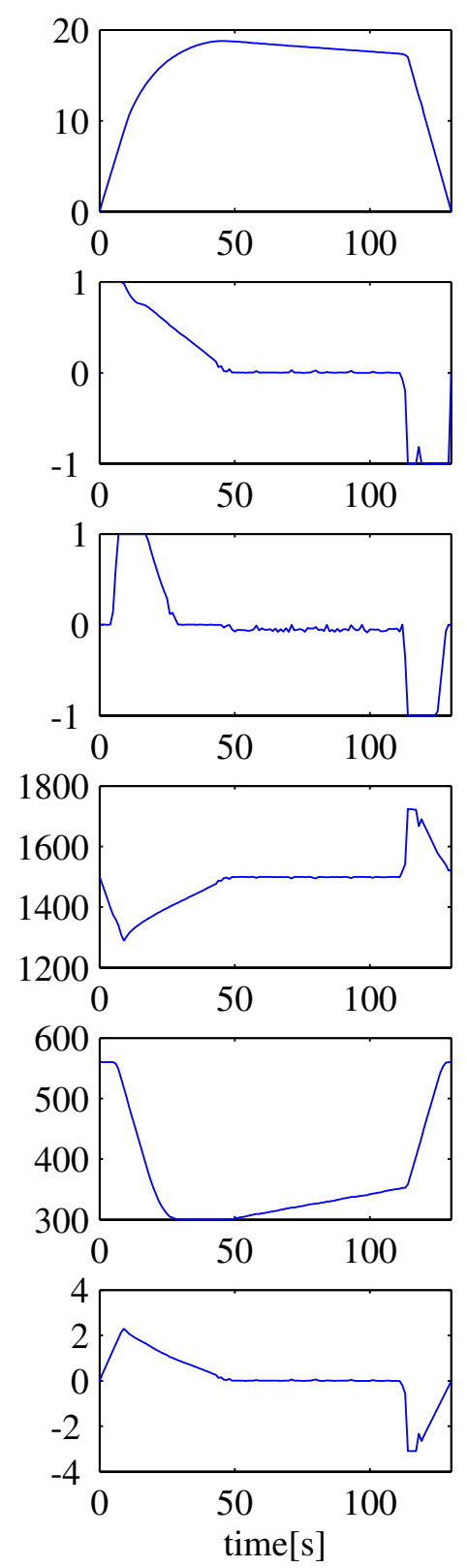

(b)Case 3

Figure 5: Optimization results. 


\section{Conclusion}

This paper presents the optimal train operation with EDLC minimizing energy consumption. SQP can be applied to the formulated optimal control problem with discrete-time transformation. As a result, it is found that the energy consumption supplied from substations can be reduced by using on-board energy storage device effectively. Compared with the train without EDLC, the total energy consumption is reduced by $0.35 \%$ and $2.92 \%$ in Case 1 and Case 2 respectively. It is also clarified that the EDLC should not fully discharge when the internal resistance is high.

A further direction of this study will be to optimize the train operation problem which has more complicated running conditions, for example, speed limitations and gradients.

\section{References}

[1] H. Ko, T. Koseki and M. Miyatake: "Numerical Study on Dynamic Programming Applied to Optimization of Running Profile of a Train", IEEJ Transactions on Industry Applications, Vol.125-D, No.12, pp. 1084-1092, 2005 (in Japanese).

[2] H. Ko, T. Koseki and M. Miyatake, "Application of Dynamic Programming to Optimization of Running Profile of A Train", Computers in Railways, WIT Press, pp. 103-112, 2004.

[3] Imad M. Nejdawi, Kevin A. Clements and Paul W. Davis, "an efficient interior point method for sequential quadratic programming based optimal power flow", IEEE Trans. on Power Systems, vol.15, NO.4, November 2000, pp. 1179-1183.

[4] G. Irisarri, L. M. Kimball, K. A. Clements, A. Bagchi and P. W. Davis, "Economic Dispatch with Network and Ramping Constraints via Interior Point Methods" IEEE Trans. on Power Systems, Vol.13, No.1, February 1998 